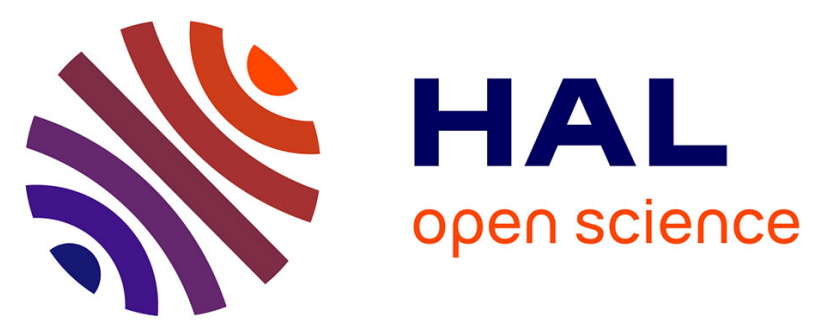

\title{
Translational Inelasticity of Hydrogen Atoms Scattering off Hydrogen-Covered W(110) Surfaces
}

Raidel Martin Barrios, Oihana Galparsoro, Aliezer Martínez Mesa, Llinersy Uranga-Piña, Cédric Crespos, Pascal Larregaray

\section{- To cite this version:}

Raidel Martin Barrios, Oihana Galparsoro, Aliezer Martínez Mesa, Llinersy Uranga-Piña, Cédric Crespos, et al.. Translational Inelasticity of Hydrogen Atoms Scattering off Hydrogen-Covered W(110) Surfaces. Journal of Physical Chemistry C, 2021, 125 (25), pp.14075-14081. 10.1021/acs.jpcc.1c03211 . hal-03398784

\section{HAL Id: hal-03398784 \\ https://hal.science/hal-03398784}

Submitted on 23 Oct 2021

HAL is a multi-disciplinary open access archive for the deposit and dissemination of scientific research documents, whether they are published or not. The documents may come from teaching and research institutions in France or abroad, or from public or private research centers.
L'archive ouverte pluridisciplinaire HAL, est destinée au dépôt et à la diffusion de documents scientifiques de niveau recherche, publiés ou non, émanant des établissements d'enseignement et de recherche français ou étrangers, des laboratoires publics ou privés. 


\title{
Translational Inelasticity of Hydrogen Atoms Scattering off Hydrogen-covered W(110) Surfaces
}

\author{
Raidel Martin Barrios, ${ }^{*},+, \neq \uparrow$ Oihana Galparsoro,, , $\|$ Aliezer Martínez Mesa, \\ Llinersy Uranga-Piña, ${ }^{\dagger, \uparrow}$ Cédric Crespos, ${ }^{\dagger, \ddagger}$ and Pascal Larregaray ${ }^{*, \dagger, \ddagger}$ \\ †CNRS, ISM, UMR5255, F-33400 Talence, France. \\ $\ddagger$ Univ. Bordeaux, ISM, UMR5255, F-33400 Talence, France. \\ IDynamical processes in Atomic and Molecular Systems (DynAMoS), Facultad de Física, \\ Universidad de la Habana, San Lázaro y L, La Habana 10400, Cuba. \\ $\S$ Georg-August Universität Göttingen, Institut für Physikalische Chemie,Tammannstraße 6, \\ 37077 Göttingen, Germany \\ ||Department of Dynamics at Surfaces, Max-Planck-Institute for Biophysical Chemistry, \\ Am Faßberg 11, 37077 Göttingen, Germany \\ E-mail: rmartin@fisica.uh.cu; pascal.larregaray@u-bordeaux.fr
}

\begin{abstract}
Quasi-classical trajectory simulations are performed to investigate the energy transfer upon scattering of hydrogen atoms off hydrogen-covered (110) tungsten surfaces. For hydrogen atoms impinging metal surfaces at energies of the order of few electronvolts, translation energy loss has been recently demonstrated to be mainly mediated by electron-hole pair excitations. The present theoretical approach scrutinizes the effect of coverage by hydrogen pre-adsorbed atoms on this energy loss. Scattering can
\end{abstract}


be rationalized in terms of three distinct dynamical mechanisms, the contribution of which changes with coverage. These allow in particular to understand why the energy loss spectra are critically affected depending on whether scattering is analysed in the whole space or at specular angle.

\section{Introduction}

Due to their prominent role in phenomena of natural and technological relevance, elementary chemical processes at surfaces have been intensively investigated both experimentally and theoretically for decades. ${ }^{1,2}$ Nevertheless, the simplest heterogeneous reactions are still of great interest. As an example, the very fundamentals hydrogen $(\mathrm{H})$ atom adsorption on metals, have been under intense scrutiny in the last five years. ${ }^{3-7} \mathrm{H}$ adsorption is indeed of importance to many fields, from interstellar molecular hydrogen production ${ }^{8}$ to heterogeneous catalysis, ${ }^{9}$ stabilization of intrinsically reactive solids, ${ }^{10}$ hydrogen storage technologies ${ }^{11,12}$ or plasma-wall interactions within the context of nuclear fusion. ${ }^{13-17}$

Atomic adsorption on a surface implies that the atom relaxes, upon collision, by dissipating its initial translational energy and that of the chemical bond to be formed with the solid. These might add up to several eV. Recent state-of-the-art works, combining atomic beam scattering experiments and molecular dynamics simulations ${ }^{3,4}$ have shown that, for $\mathrm{H}$ scattering, inelastic energy losses are very similar for a series of six fcc metal (111) surfaces. ${ }^{4}$ Charge transfer between hydrogen and the surface can be discarded and, though a minor fraction of energy transfer occurs via couplings to phonons, energy dissipation is shown to be mainly governed by electron hole pair (ehp) excitations. Indeed, the experiments measuring $^{3,4}$ the translational energy of $\mathrm{H}$ atoms scattered off the metals could only be rationalized by accounting for electronic excitations of metal electrons in molecular dynamics simulations. Besides, studies on $\mathrm{H}_{2}$ diatom recombination by collision of $\mathrm{H}$ atoms on $\mathrm{H}$-covered Tungsten surfaces have also highlighted the major influence of ehp excitations for slowing down and stopping Hot-Atoms initially diffusing at hyperthermal velocities on surfaces. ${ }^{18,19}$ 
To the best of our knowledge, the translation energy loss of $\mathrm{H}$ atom scattering off surfaces have only been measured and rationalized for clean metal surfaces. However, as H adsorption on metal is a very efficient process, the question which then arises is : how is the energy transfer to the surface affected when surface gets populated with adsorbates? This is the question we intend to address in details in the present work.

To do so, we focus on the theoretical description of translation inelasticity upon collision of $\mathrm{H}$ with pristine and H-covered (110) tungsten (W) surfaces. The H-W system is chosen here for its current relevance, as tungsten is a promising candidate to constitute the divertors of the coming ITER nuclear fusion reactor. ${ }^{13-17}$ Here, we use quasi-classical trajectory simulations including electronic friction relying on a previously developed ground state potential energy surface. The approach is quite similar to the one which was used in previous work to rationalize translational inleasticity on clean metals. ${ }^{4}$

The remaining of the paper is organized as follows. The methodology and details of the calculations are presented in section 2. In section 3, the results are shown and discussed. Finally, section 4 concludes.

\section{Theoretical methods}

Quasi-classical trajectory (QCT) simulations have been carried out for the pristine $(\Theta=0)$ and covered $(\Theta=0.25,0.75 \mathrm{ML}) \mathrm{W}(110)$ surfaces. The approach relies on a Density Functional Theory (DFT) based multiadsorbate potential energy surface (PES) ${ }^{18-20}$ previously developed from an adaptation of the corrugation reducing procedure $(\mathrm{CRP})^{21,22}$ within the frozen surface approximation. Such an approximation is based on the large H/W mass mismatch $(1 / 183.5)$, which is expected to lead to minor energy transfers to tungsten phonons. ${ }^{3,4}$ The necessary DFT calculations were performed using the slab supercell approach and within the generalized gradient approximation PW91 functional ${ }^{23,24}$ to describe electronic exchange and correlation. As previously described, ${ }^{18,25}$ the finite coverage potential is developed as a 
two-H terms expansion, i.e.,

$$
V\left(\left\{\mathbf{r}_{i}\right\}\right)=\sum_{i=1}^{N} V^{3 D}\left(\mathbf{r}_{i}\right)+\sum_{i=1}^{N} \sum_{j>i}^{N} I^{6 D}\left(\mathbf{r}_{i}, \mathbf{r}_{j}\right),
$$

where $\mathbf{r}_{i}$ is the position vector of atom $i, V^{3 D}\left(\mathbf{r}_{i}\right)$ is the 3-dimensional $\mathrm{H} / \mathrm{W}(110)$ interaction potential, and $I^{6 D}\left(\mathbf{r}_{i}, \mathbf{r}_{j}\right)$ is the six-dimensional diatomic interpolation function. ${ }^{20,21}$ The PES describe $\mathrm{H}$ subsurface penetration down to $-4.4 \AA(Z=0$ is defined by the altitude of the topmost surface layer). A thorough comparison with DFT calculations has been performed elsewhere $^{20}$ evidencing that the validity of the multiadsorbate PES to accurately describe the surface $H$ arrangements and energetics up to $\Theta=1 \mathrm{ML}$.
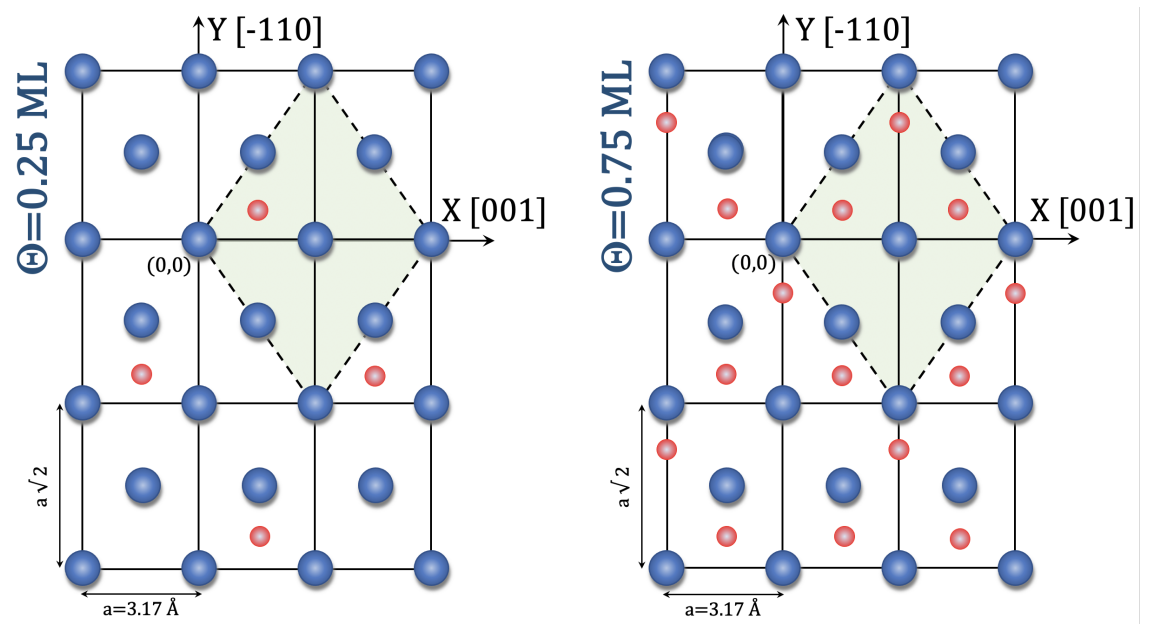

Figure 1: Position of the adsorbed $\mathrm{H}$ atoms (red points) at $\Theta=0.25 \mathrm{ML}$ (left) and $\Theta=0.75$ $\mathrm{ML}$ (right). The lattice constant parameter is $\mathrm{a}=3.17 \AA$. Sampling areas of initial $\left(\mathrm{X}_{p}, \mathrm{Y}_{p}\right)$ positions of the projectiles for each coverage are represented in green. Details about the energetics and structure of the adsorbed layer can be found in ref. ${ }^{25}$

The present QCT calculations use a $6 \times 6$ rectangular $(a \times a \sqrt{2})$ supercell with periodic boundary conditions in order to model an infinite covered surface. The classical equations of motion are integrated for one projectile atom and 18 (54) adsorbed targets for $\Theta=0.25$ ML (0.75 ML) coverages respectively. The H-adsorbates initially sit in their equilibrium positions (close to the threefold hollow site) and they are given the Zero Point Energy (ZPE). The positions of the adsorbates are schematized in Fig. 1. Details about the adsorbed 
layer structure, energetics and the semiclassical procedure to ascribe the ZPE can be found elsewhere. ${ }^{18-20}$ The coordinate system originates on top of a tungsten atom. The X, Y, Z axes lie respectively along the [001], [-110] and [110] crystal directions.

The initial altitude of the projectile is taken in the asymptotic region of the potential, at $Z_{p}=7.0 \AA$ from the surface. The $\left(\mathrm{X}_{p}, \mathrm{Y}_{p}\right)$ initial position of the projectile is randomly sampled in the covered surface irreducible unit cell (green areas of Figure 1). In order to perform simulations using conditions of experimental relevance, ${ }^{3} \mathrm{H}$ atoms are sent with $E_{i}=2.75 \mathrm{eV}$ collision energy and $\theta_{i}=45^{\circ}$ polar angle (with respect to the Z[110] surface normal) and $\phi_{i}=0^{\circ}$ azimuthal angle (with respect to the X[001] axis) incidence. Scattered atoms are analysed either in the whole space or at specular $\left(\theta_{\text {scat }}=45 \pm 5^{\circ}, \phi_{\text {scat }}=0 \pm 2^{\circ}\right)$ scattering angle. To ensure numerical convergence, more than $2 * 10^{6}$ trajectories have been run at each coverage. Since the multiadsorbate PES ignores three-body and higher-order contributions to the interaction between hydrogen atoms (see eq. 1), trajectories are discarded whenever one $\mathrm{H}$ atom has two neighbouring $\mathrm{H}$ atoms closer than $1.5 \AA$. The number of such trajectories in not significant when analyzing atom scattering (respectively lower than $0.05 \%$ and $2.4 \%$ for $\Theta=0.25$ and $0.75 \mathrm{ML})$.

Metal electron excitations (ehp) are taken into account within the Local Density Friction Approximation (LDFA). ${ }^{26,27}$ Within this model, electronic nonadiabaticity is introduced through dissipative and random forces in the classical equations of motion for the hydrogen atoms (Langevin equation, see eq. 2).

$$
m \ddot{\mathbf{r}} i=-\frac{\partial V\left(\left\{\mathbf{r}_{i}\right\}\right)}{\partial \mathbf{r}_{i}}-\eta_{e l}\left(\mathbf{r}_{i}\right) \dot{\mathbf{r}}_{i}+\mathbf{F}_{\mathbf{L}}(\mathbf{t}),
$$

where $m$ is the mass of hydrogen. The friction force, proportional to the atom velocity, depends on the vector position, $\mathbf{r}_{i}$, of the atom $i$, through the friction coefficient, $\eta_{e l}\left(\mathbf{r}_{i}\right)$. Within the LDFA, $\eta_{e l}\left(\mathbf{r}_{i}\right)$ is taken as that of the same atom moving in a homogeneous free electron gas with electronic density equal to that of the bare surface at the same position 
$\mathbf{r}_{i} \cdot 3,5,26,27$ The random force, $\mathbf{F}_{\mathbf{L}}(\mathbf{t})$, related to the friction force by the second fluctuationdissipation theorem, accounts for electron temperature and is taken here as a Gaussian white noise. ${ }^{27}$ In the present calculations, electronic temperature is set at $\mathrm{Ts}=300 \mathrm{~K}$. The random force term has often been neglected when surface electron temperature is not high, as in the works rationalizing the relaxation of $\mathrm{H}$ hot-atoms on bare metallic surface, ${ }^{28-30}$ or diatom recombination. ${ }^{18,19,31}$ However, this term is crucial when elementary surface processes are triggered by intense laser pulses causing electrons to be heated up to a few thousands of Kelvin. ${ }^{27,32-38}$ Recently, this term has been also shown to sensibly alter the shape of specular energy loss spectra of hydrogen scattering from clean metal surfaces ${ }^{39}$ even at ambient temperatures.

Dissipation to surface phonons is here neglected on the ground that, as recently demonstrated, ${ }^{3,4,28,29,40-42}$ dissipation to electrons largely dominates the relaxation of hydrogen on metals at short time scales. To prevent leakage of the ZPE, the friction acts only when the energy of the pre-adsorbed atom exceeds the ZPE. Details can be found in refs. ${ }^{18,19}$

Several elementary processes may occur upon a H-atom collision: reflection (or atomic scattering) whenever the impinging atom is reflected back to the initial altitude, abstraction if a $\mathrm{H}_{2}$ molecule reaches this altitude, absorption if one atom lies below the surface $(Z<0 \AA)$ after the 1 ps total integration time and otherwise adsorption. It has been checked that a longer integration time does not modify the results. Adsorption and reflection are the most probable outcomes from hydrogen collision at the incidence of interest. Adsorption probabilities are $0.28,0.37$ and 0.48 at $0,0.25$ and $0.75 \mathrm{ML}$ coverages, for which reflection probabilities are $0.69,0.58$ and 0.38 , respectively. These opposite trends highlight the efficient energy exchange of energy between the incoming $\mathrm{H}$ atom and the adsorbed ones. Absorption probabilities are low (respectively 0.03, 0.026 and 0.0154) and decrease with coverage as adsorbed atoms block the possible paths into the bulk. Abtraction is a low probability channel which consistently increases with coverage, being respectively 0.026 and 0.079 at 0.25 and $0.75 \mathrm{ML}$ coverages. In the following, we will focus on the reflection (or 


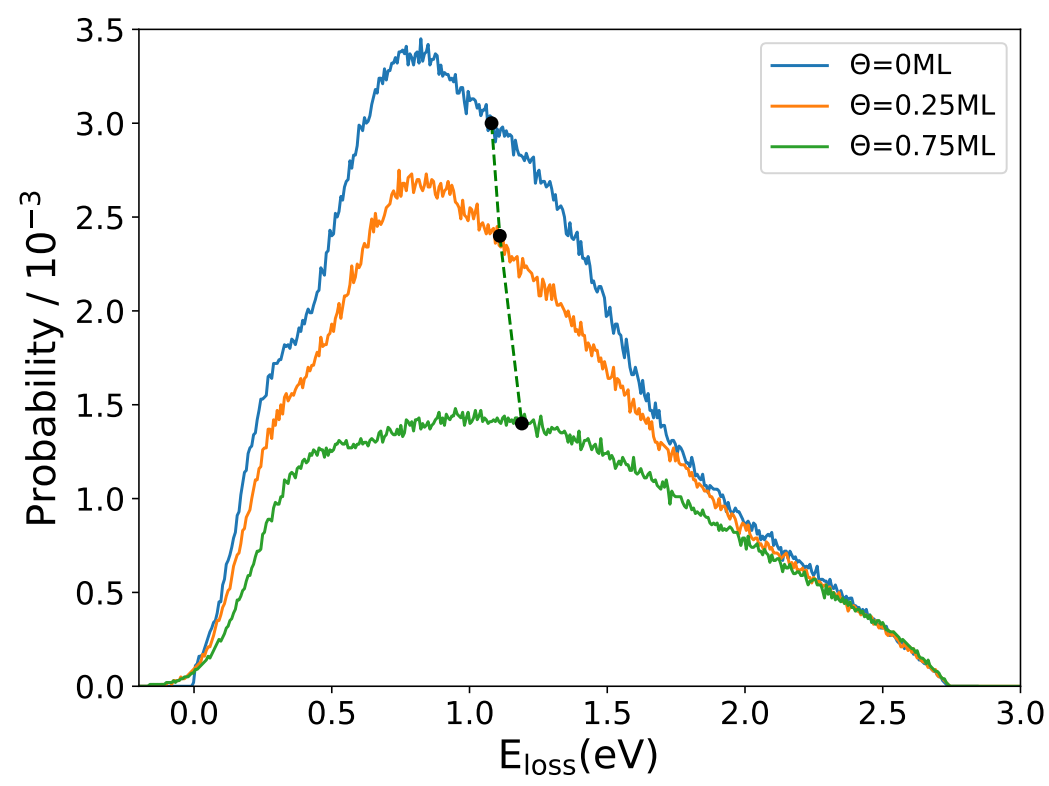

Figure 2: Energy loss spectra for all scattered atoms, for $\Theta=0$ (blue), 0.25 (orange) and 0.75 (green) ML. The initial conditions for the simulations are $E_{i}=2.75 \mathrm{eV}, \phi_{i}=0^{\circ}, \theta_{i}=45^{\circ}$ and $\mathrm{Ts}=300 \mathrm{~K}$. The distributions are normalized to the total number of trajectories. Black circles represent the average energy loss.

atomic scattering ) channel. We here ignore the minoritary channel in which the impinging atom is adsorbed while one initially adsorbed atom is desorbed, which was found to be less than $1.29 \%$ and $3.82 \%$ of the scattered trajectories for respectively $\Theta=0.25$ and 0.75 ML.

\section{Results and Discussion}

The kinetic energy loss spectra for all scattered $H$ atoms off clean $(\Theta=0)$ and H-covered $\mathrm{W}(110)$ surfaces $(\Theta=0.25,0.75)$ are displayed in Fig. 2. The spectra are normalized to the total number of trajectories so that the area is proportional to the total scattering probability. As mentioned above, this probability decreases with increasing coverage, being 0.69, 0.58 and 0.38 for respectively $0,0.25$ and $0.75 \mathrm{ML}$ coverages. This evolution reflects the ability of the $\mathrm{H}$-adsorbates to efficiently dissipate the kinetic energy of the impinging $\mathrm{H}$ atoms. For the clean surface, electron-hole pair excitation is the only dissipation channel accounted for 


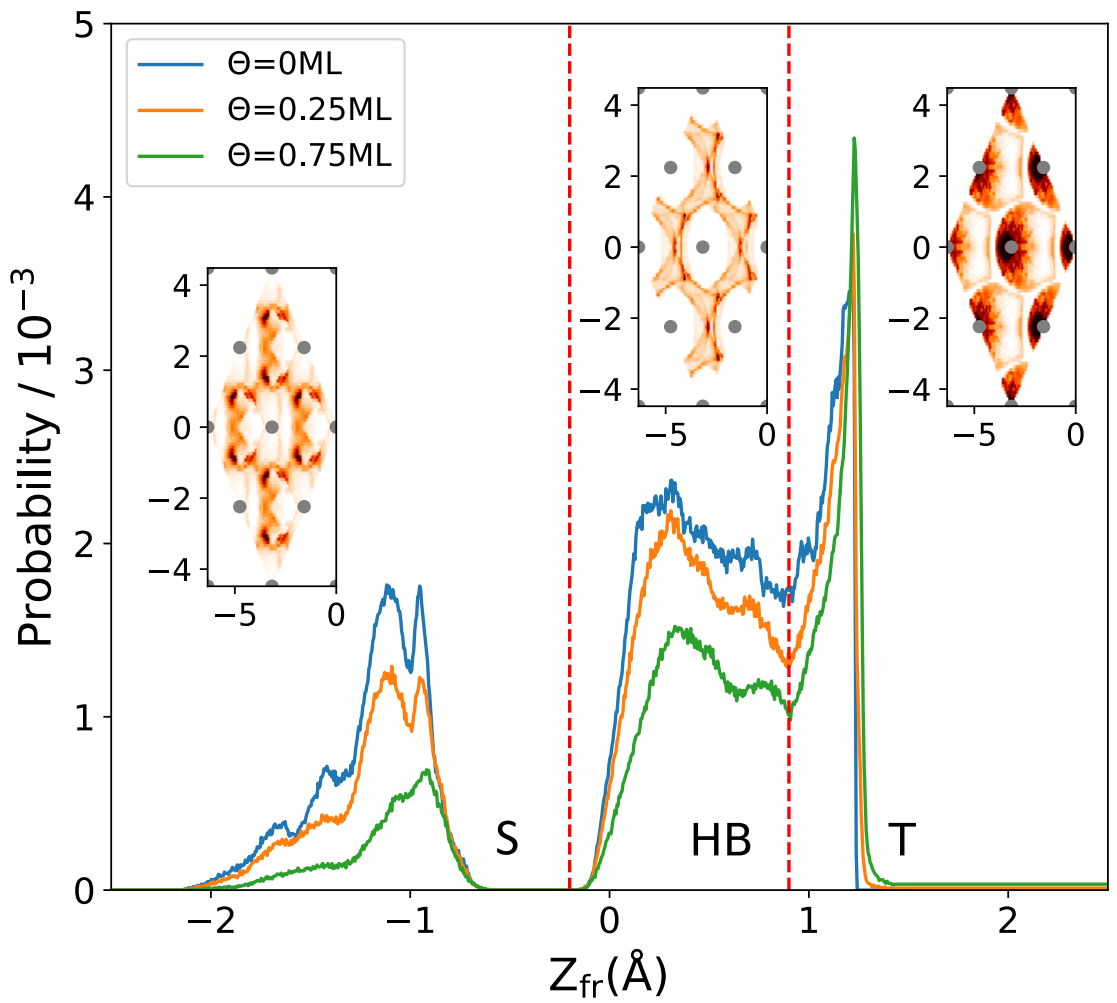

Figure 3: Distribution of the projectile first rebound altitudes, $Z_{f r}$, for $\Theta=0$ (blue), 0.25 (orange) and 0.75 (green) ML. T-contribution corresponds to a narrow peak $\left(Z_{f r}>0.8 \AA\right)$, HB-contribution corresponds to a broad peak $\left(-0.2 \AA>Z_{f_{r}}>0.8 \AA\right)$, and S-contribution corresponds to a broad peak $\left(-2.1 \AA>Z_{f r}>-0.2 \AA\right)$. For the three contributions, the $(\mathrm{X}, \mathrm{Y})$ distributions of the projectile first rebound are displayed in the insets ( $\AA$ units), at zero coverage. The darker the color, the higher reflection probability. The grey points represent the $\mathrm{W}$ surface atoms.

in the simulation. At finite coverages, collisions with adsorbed species efficiently remove incidence kinetic energy from the projectile to lead to adsorption as illustrated in recent works on recombination at surfaces. ${ }^{18,19}$ At zero coverage, the energy loss spectrum shows a pronounced peak around $0.75 \mathrm{eV}$ with two shoulders at lower $(0.25 \mathrm{eV})$ and higher $(1.25 \mathrm{eV})$ energies. These structures tend to disappear with increasing coverage. The average energy loss slightly increases with coverage, being $1.08,1.11$ and $1.19 \mathrm{eV}$ at respectively $0,0.25$ and 0.75 ML coverages. The corresponding average relative energy losses are 0.39, 0.4 and 0.43. The value for the clean surface is in good agreement with experiment on other metals. ${ }^{4}$

The energy loss spectra can be qualitatively described in terms of the contributions 
of three subsets of trajectories involving distinct scattering dynamics. Fig. 3 displays the distributions of the H-projectile first rebound altitudes $\left(Z_{f r}\right)$ for the three coverages. A rebound is here defined as the sign change of the momentum Z-component (first turning point along the surface normal). The insets display the distribution of the (X,Y) rebound positions, for the clean surface, for the above mentioned subsets of scattering events. The first one, T-scattering (T stands for Top), corresponds to a narrow peak above $Z>0.8 \AA$ for which the first rebounds take place on top of tungsten surface atoms (circles in the insets). For the second subset, HB-scattering (H and B stand for Hollow and Bridge, respectively), the rebound altitude distribution corresponds to a broad peak $(-0.2 \AA<Z<0.8 \AA)$ and the projectiles first bounce between the tungsten surface atoms without penetrating the bulk. For the third one, S-scattering (S stands for Subsurface), the rebound altitude distribution also corresponds to a broad peak $(-2.1 \AA<Z<-0.2 \AA)$ but the projectile penetrates below the surface to bounce off second layer tungsten atoms lying below the four-fold hollow symmetry surface sites at $Z=-2.24 \AA$. As apparent from the rebound altitude distribution, the three subsets show up whatever the coverage considered in this work. 


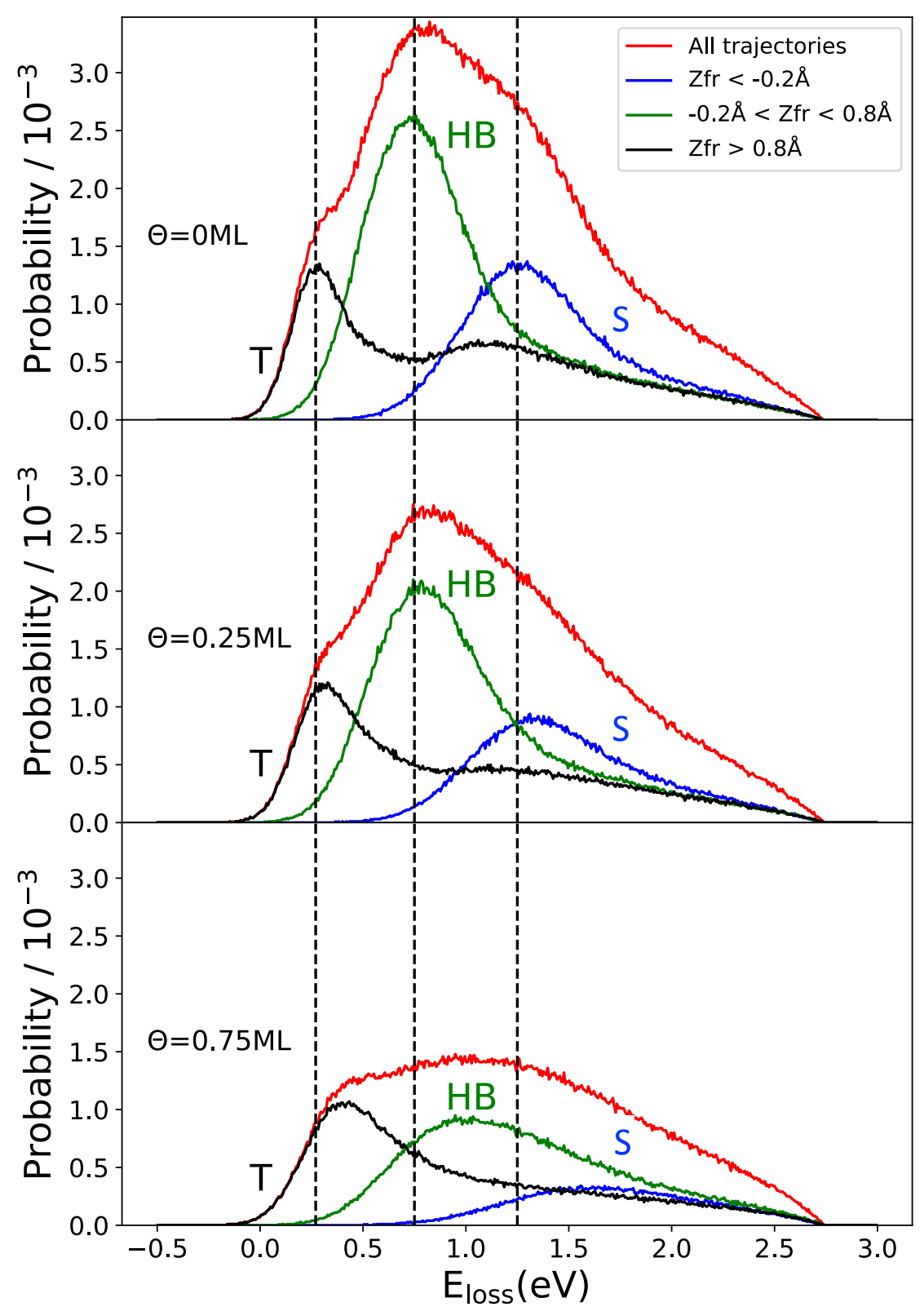

Figure 4: Energy loss spectra for all scattered atoms (red) decomposed into the T-(black), HB-(green) and S-(blue) contributions for $\Theta=0$ (top), 0.25(middle) and 0.75 ML (down). The vertical dashed lines indicate the maxima at 0 coverage.

Fig. 4 displays the contributions of each subsets to the kinetic energy loss spectra of Fig. 2. For the clean surface (upper panel), the energy loss spectrum of each component consists of a prominent peak developing from the lowest energy loss, followed by a slowly decaying tail. These prominent peaks are separate by approximately $0.5 \mathrm{eV}$. For the Tcomponent, a secondary maximum even show up. Theses tails correspond to trajectories 
reflecting back to the gas phase after bouncing on the surface more than once (see supporting information). Reasonably, at 0 coverage, there is a correlation between the altitude of the rebound and the amount of energy loss. The S-scattering component, which involves sub-surface scattering, loses on average more energy than the HB-component, bouncing in between tungsten surface atoms, which loses itself more than the T-component for which H-atoms bounce on top on surface atoms. Within the LDFA, the energy loss increases when the electronic density experienced along the trajectory increases. Thus, the closest the projectile gets to/into the surface, the higher the electron density and the amount of energy transferred to the substrate. It can be seen that the energy loss spectrum of each component is displaced towards higher energies as coverage increases because of interaction with adsorbates. Moreover, the scattering probabilities for the HB- and S-contributions are strongly reduced by coverage. The T-, HB- and S-scattering probabilities lowers from respectively $0.196,0.32$ and 0.182 for the clean surface to $0.16,0.17$ and 0.06 at $0.75 \mathrm{ML}$ coverage. Consequently, the relative contribution for T-scattering increases from $28 \%$ to $41 \%$. This evolution is to be expected as the dynamics of HB- and S-scattering involve motions in-between tungsten atoms, i.e. possibly close to where the adsorbed $\mathrm{H}$-atoms sit (three-fold hollow site). This leads to a much stronger interaction with adsorbates for HBand S-scattering, thus favoring adsorption.

To summarize, as far as all the reflections are concerned, the average energy loss and adsorption probability increase with coverage, what can be intuitively rationalized in terms of the interaction with the adsorbates, as the impinging atom undergoes efficient energy exchange with them. 


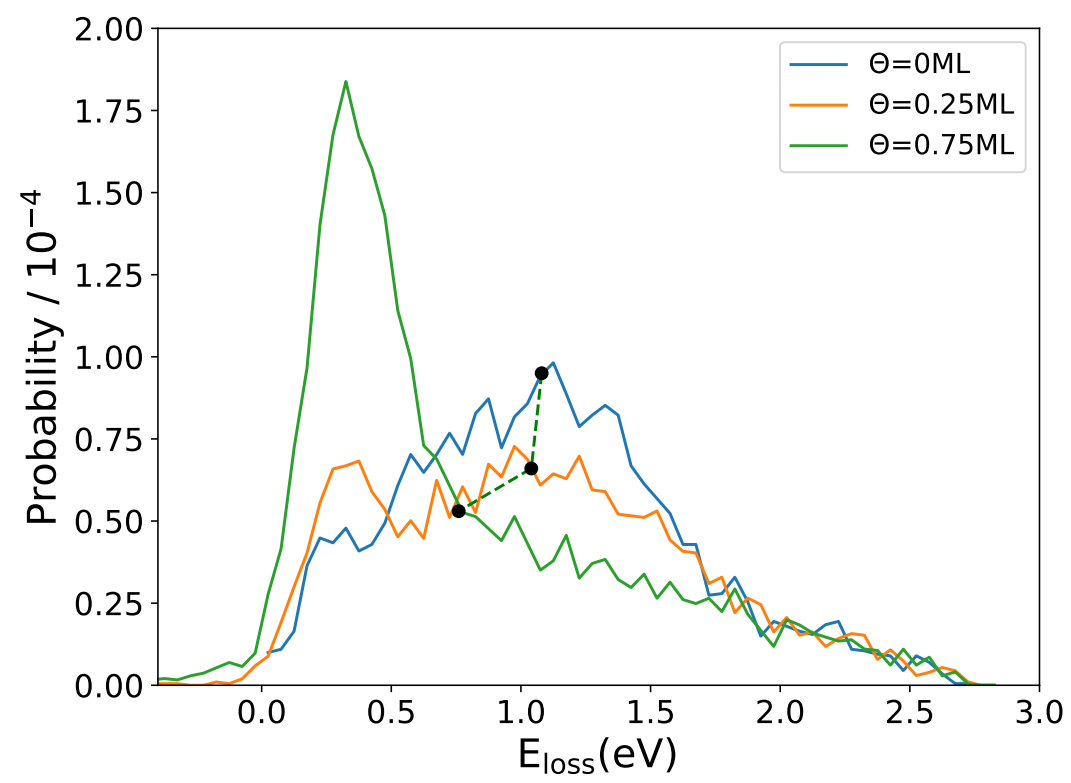

Figure 5: Energy loss spectra for atoms scattered at specular angle, for $\Theta=0$ (blue), 0.25 (orange) and 0.75 (green) ML. The distributions are normalized to the total number of trajectories. Black circles represent the average energy loss.

Nevertheless, most experiments do not measure scattering into the whole space but rather at specific scattering angles. ${ }^{3,4}$ Fig. 5 displays the energy loss spectra at the specular angle $\theta_{\text {scat }}=45^{\circ}, \phi_{\text {scat }}=0^{\circ}$. Contrary to what is observed for all scattering events (see Fig. 2), the shape of the energy loss spectra importantly changes and the energy loss decreases when coverage increases. This trend can be rationalized in terms of the three subsets of scattering events. Fig. 6 displays the contribution of the T-, HB- and S-scattering to the specular energy loss spectra. It is apparent from the figure that contributions of HB- and S-scattering sensibly decrease for increasing coverage. Again, interaction with the adsorbates might either deviate the projectile from the specular direction or exchange sufficient energy to lead to ensuing adsorption. 


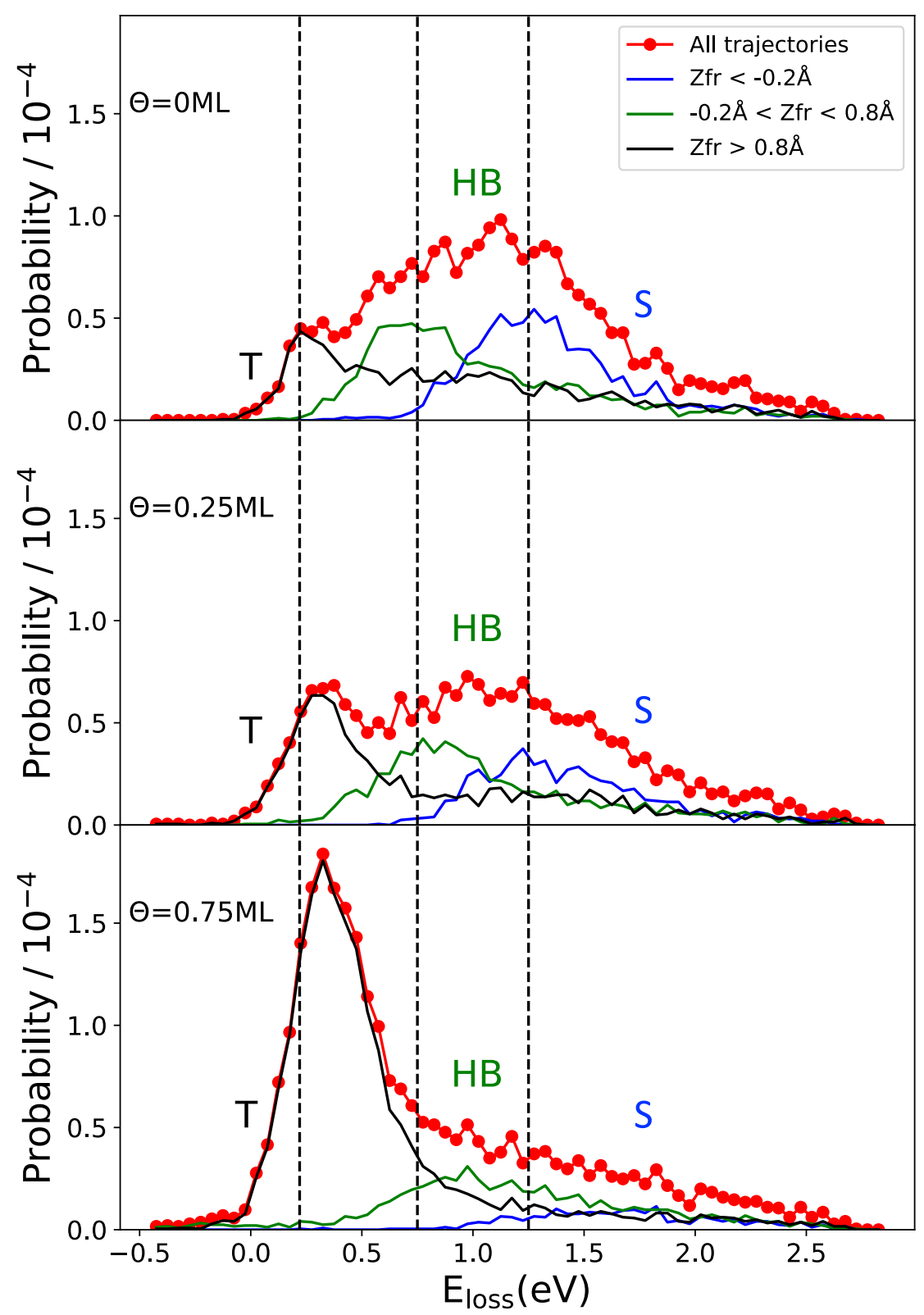

Figure 6: Energy loss spectra for atoms scattered at specular angle (red) decomposed into the T-(black), HB-(green) and S-(blue) contributions for $\Theta=0$ (top), 0.25(middle) and 0.75 ML (down). The vertical dashed lines indicate the maxima at 0 coverage.

On the contrary, the contribution of T-scattering increases importantly suggesting that specular scattering is favored by coverage for projectile scattering off top tungsten atoms. This is indeed confirmed by Fig. 7 which presents the evolution of the distribution of the polar and azimuthal scattering angles for the T-scattering component as a function of coverage. Specular scattering is markedly favored. Thus, when restricting to the specular angle, 
the contribution of scattering on top of tungsten atoms is amplified. This lead to the counterintuitive result that increasing coverage of Tungsten with H-adsorbates lowers the energy loss of $\mathrm{H}$ atoms.

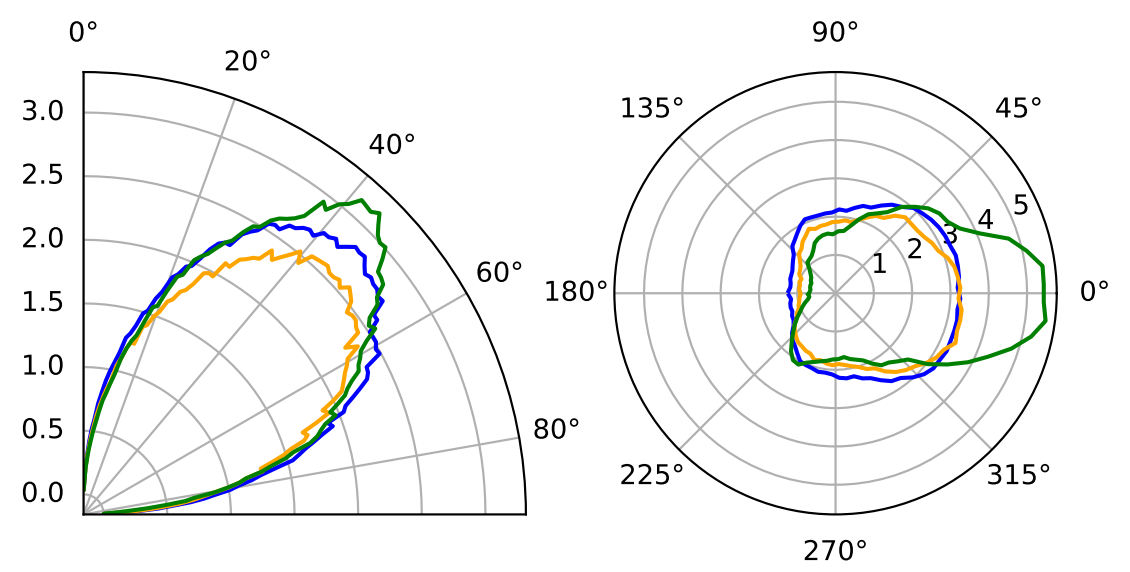

Figure 7: Distribution of the polar (left) and azimuthal (right) scattering angles of reflected $\mathrm{H}$ atoms upon T-scattering for $\Theta=0$ (blue), 0.25 (orange) and $0.75 \mathrm{ML}$ (green) coverage.

\section{Conclusions}

The quasi-classical trajectory method has been applied to the study of hydrogen atom scattering off clean and H-covered (110) tungsten surface, and accounting for electron-hole pair excitation of metal electrons. The QCT simulations provided a clear, mechanistic picture of energy loss spectra of the scattered atoms.

When considering total reflection, the scattering probability decreases with increasing coverage, due to the very efficient energy transfer via binary collision between impinging and pre-adsorbed atoms, leading to adsorption. The average energy loss of the scattered atoms consistently increases.

However, when specular reflection is only considered, the opposite effect is observed, i.e. the average energy loss decreases with increasing coverage. This effect can be rationalized in terms of the dynamics of the hydrogen surface collision. On the one hand, at zero coverage, atoms scattered at the specular angle mainly result from collision at the bridge and hollow 
sites, with a sizeable proportion ongoing subsurface penetration before reflection. Such events lead to an important loss of energy by electron-hole pair excitations as high surface electronic densities are experienced by the impinging $\mathrm{H}$ atoms. On the other hand, when coverage is high $(\Theta=0.75 \mathrm{ML})$, atoms scattered at the specular angle originate mainly from collisions on top of tungsten atom surface sites. Such events correspond to a minimal energy loss as hydrogen atoms bounce relatively far from the surface (above $0.8 \AA$ ), thus minimizing electron-hole pair excitations, and far from the pre-adsorbed hydrogen atoms sitting close to the three-fold hollow sites. Atoms bouncing close to the bridge and hollow sites conversely interacts strongly with the adsorbates and are thus deflected away the specular angle or eventually adsorb on the surface.

The analysis of scattering at the specular angle thus lead the counterintuitive result that increasing coverage of Tungsten with hydrogen adsorbates lowers the energy loss of impinging $\mathrm{H}$ atoms.

\section{Notes}

Conflict of interest: None

Authors Contributions: RMB and PL contributed equally to the paper. OGL, AMM, LUP and CC mainly contribute to reading and correcting.

\section{Supporting Information}

Influence of the number of rebounds on energy loss spectra 


\section{Acknowledgements}

The authors acknowledge the support of the French Embassy in Cuba, the University of Bordeaux, the CNRS, the Erasmus Mundus program for funding and ISM and University of Bordeaux for providing computing resources. This work was conducted in the scope of the transborder joint Laboratory QuantumChemPhys: Theoretical Chemistry and Physics at the Quantum Scale (ANR-10-IDEX-03-02)

\section{TOC entry}

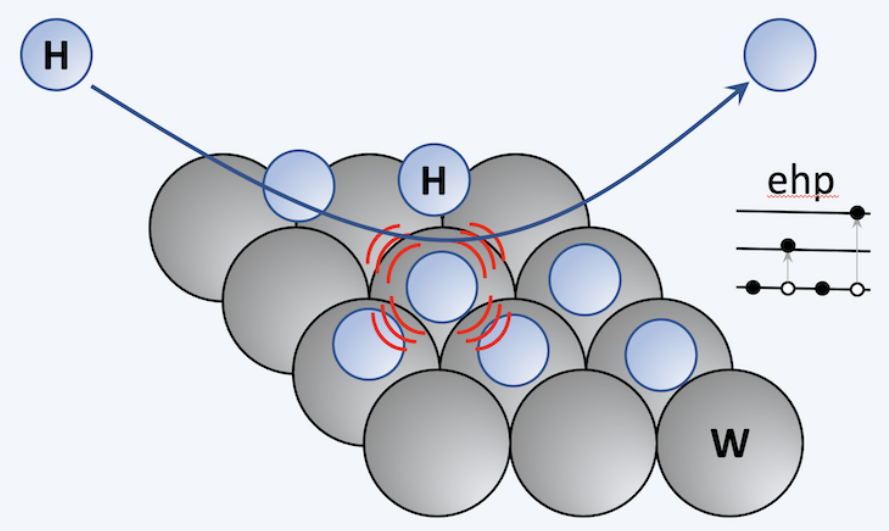

\section{References}

(1) Díez Muiño, R.; Busnengo, H. Dynamics of Gas-Surface Interactions: Atomic-level Understanding of Scattering Processes at Surfaces; Springer Series in Surface Sciences; Springer, 2013.

(2) Somorjai, G. A.; Li, Y. Introduction to surface chemistry and catalysis; John Wiley \& Sons, 2010.

(3) Bünermann, O.; Jiang, H.; Dorenkamp, Y.; Kandratsenka, A.; Janke, S. M.; Auer- 
bach, D. J.; Wodtke, A. M. Electron-hole pair excitation determines the mechanism of hydrogen atom adsorption. Science 2015, 350, 1346-1349.

(4) Dorenkamp, Y.; Jiang, H.; Köckert, H.; Janke, A., Svenja Mand Kandratsenka; Wodtke, A. M.; Bünermann, O. Hydrogen collision with transition metal surfaces: Universal electronically non adiabatic adsorption. The Journal of Chemical Physics 2018, $148,034706$.

(5) Janke, S. M.; Auerbach, D. J.; Wodtke, A. M.; Kandratsenka, A. An accurate fulldimensional potential energy surface for H-Au(111): Importance of nonadiabatic electronic excitation in energy transfer and adsoroption. The journal of Chemical Physics 2018, 143, 124708 .

(6) Kammler, M.; Janke, S. M.; ; Kandratsenka, A.; Wodtke, A. M. Genetic Algorithm approach to global optimization of the full-dimensional potential energy surface for hydrogen atom at fcc-metal surfaces. Chemical Physics Letter 2017, 683, 286-290.

(7) Kandratsenka, A.; Jiang, H.; Dorenkamp, Y.; Janke, S. M.; Kammler, M.; Wodtke, A. M.; Bünermann, O. Unified description of H-atom-induced chemicurrents and inelastic scattering. Proceedings of the National Academy of Sciences 2018, $\underline{115}$, $680-684$.

(8) Hollenbach, D.; Salpeter, E. Surface recombination of hydrogen molecules. The Astrophysical Journal 1971, 163, 155.

(9) Andersson, M.; Abild-Pedersen, F.; Remediakis, I.; Bligaard, T.; Jones, G.; Engbæk, J.; Lytken, O.; Horch, S.; Nielsen, J. H.; Sehested, J., et al. Structure sensitivity of the methanation reaction: H2-induced CO dissociation on nickel surfaces. Journal of

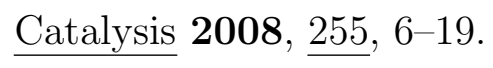

(10) Higashi, G.; Chabal, Y.; Trucks, G.; Raghavachari, K. Ideal hydrogen termination of the Si (111) surface. Applied physics letters 1990, 56, 656-658. 
(11) Sakintuna, B.; Lamari-Darkrim, F.; Hirscher, M. Metal hydride materials for solid hydrogen storage: a review. International journal of hydrogen energy 2007, 32, 11211140.

(12) Lee, S. M.; Park, K. S.; Choi, Y. C.; Park, Y. S.; Bok, J. M.; Bae, D. J.; Nahm, K. S.; Choi, Y. G.; Yu, S. C.; Kim, N.-g., et al. Hydrogen adsorption and storage in carbon nanotubes. Synthetic metals 2000, 113, 209-216.

(13) Kleyn, A.; Cardozo, N. L.; Samm, U. Plasma-surface interaction in the context of ITER. Physical Chemistry Chemical Physics 2006, ㅁ, 1761-1774.

(14) Barabash, V.; Federici, G.; Matera, R.; Raffray, A.; Teams, I. H. Armour materials for the ITER plasma facing components. Physica Scripta 1999, 1999, 74.

(15) Federici, G.; Wuerz, H.; Janeschitz, G.; Tivey, R. Erosion of plasma-facing components in ITER. Fusion engineering and design 2002, 61, 81-94.

(16) Federici, G.; Andrew, P.; Barabaschi, P.; Brooks, J.; Doerner, R.; Geier, A.; Herrmann, A.; Janeschitz, G.; Krieger, K.; Kukushkin, A., et al. Key ITER plasma edge and plasma-material interaction issues. Journal of Nuclear Materials 2003, 313, 11-22.

(17) Roth, J.; Tsitrone, E.; Loarte, A.; Loarer, T.; Counsell, G.; Neu, R.; Philipps, V.; Brezinsek, S.; Lehnen, M.; Coad, P., et al. Recent analysis of key plasma wall interactions issues for ITER. Journal of Nuclear Materials 2009, 390, 1-9.

(18) Galparsoro, O.; Pétuya, R.; Busnengo, H. F.; Juaristi, J. I.; Crespos, C.; Alducin, M.; Larregaray, P. Hydrogen abstraction from metal surface: when electron-hole pair excitations strongly affect hot-atom recombination. Physical Chemistry Chemical Physics 2016, 18,31378 .

(19) Galparsoro, O.; Busnengo, H. F.; Juaristi, J. I.; Crespos, C.; Alducin, M.; Larregaray, P. 
Communication: Hot-atom abstraction dynamics of hydrogen from tungsten surfaces: The role of surface structure. The journal of Chemical Physics 2017, 147, 121103.

(20) Pétuya, R.; Larrégaray, P.; Crespos, C.; Aurel, H. F., Philippe Busnengo; Martinez, A. E. Scattering of Atomic Hydrogen Off a H-Covered W(110) Surface: HotAtom versus EleyRideal Abstraction Dynamics. The Journal of Physical Chemistry C 2015, 119, 3171-3179.

(21) Busnengo, H.; Salin, A.; Dong, W. Representation of the 6D Potential Energy Surface for a Diatomic Molecule near a Solid Surface. J. Chem. Phys. 2000, 112, 7641-7651.

(22) Olsen, R. A.; Busnengo, H. F.; Salin, A.; Somers, M. F.; Kroes, G. J.; Baerends, E. J. Constructing Accurate Potential Energy Surfaces for a Diatomic Molecule Interacting with a Solid Surface: $\mathrm{H}_{2}+\mathrm{Pt}(111)$ and $\mathrm{H}_{2}+\mathrm{Cu}(100)$. J. Chem. Phys. 2002, 116, 38413855.

(23) Perdew, Y., J. P.; Wang Accurate and simple analytic representation of the electron-gas correlation energy. Phys. Rev. B 1992, 45, 13244.

(24) Perdew, J. P. Electronic Structure of Solids; Ziesche, P., Eschring, H., Eds.; AkademieVerlag: Berlin, 1991.

(25) Pétuya, R.; Larrégaray, P.; Crespos, C.; Busnengo, H. F.; Martinez, A. E. Dynamics of H2 Eley-Rideal abstraction from W (110): Sensitivity to the representation of the molecule-surface potential. The Journal of Chemical Physics 2014, 141, 024701.

(26) Juaristi, J. I.; Alducin, M.; Diéz-Muino, R.; Busnengo, H. F.; Salin, A. Role of Electron Hole Pair Excitations in the Dissociative Adsorption of Diatomic Molecules on Metal Surfaces. Physical Review Letters 2008, 100, 116102.

(27) Alducin, M.; Diéz-Muino, R.; Juaristi, J. I. Non-Adiabatic Effects in Elementary Reaction Processes at Metal Surfaces. Progress in Surface Science 2017, 92, 317-340. 
(28) Blanco-Rey, M.; Juaristi, J. I.; Díez Muiño, R.; Busnengo, H. F.; Kroes, G. J.; Alducin, M. Electronic Friction Dominates Hydrogen Hot-Atom Relaxation on Pd(100). Phys. Rev. Lett. 2014, 112, 103203.

(29) Novko, D.; Blanco-Rey, M.; Juaristi, J. I.; Alducin, M. Ab initio Molecular Dynamics with Simultaneous Electron and Phonon Excitations: Application to the Relaxation of Hot Atoms and Molecules on Metal Surfaces. Phys. Rev. B 2015, 92, 201411.

(30) Novko, D.; Blanco-Rey, M.; Juaristi, J. I.; Alducin, M. Surface Electron Density Models for Accurate Ab-Initio Molecular Dynamics with Electronic Friction. Phys. Rev. B 2016, $\underline{93}, 245435$.

(31) Galparsoro, O.; Juaristi, J. I.; Crespos, C.; Alducin, M.; Larrégaray, P. Stereodynamics of Diatom Formation through Eley-Rideal Abstraction. The Journal of Physical Chemitry C 2017, 121, 19849-19858.

(32) Saalfrank, P. Quantum Dynamical approach to ultrafast molecular desorption at surfaces. Chemical Review 2006, 106, 4116-4159.

(33) Springer, C.; Head-Gordon, M.; Tully, J. C. Simulations of femtosecond laser-induced desorption of CO from $\mathrm{Cu}(100)$. Surface Science 1994, 320, L57-L62.

(34) Springer, C.; Head-Gordon, M. Simulations of the femtosecond laser-induced desorption of $\mathrm{CO}$ from $\mathrm{Cu}(100)$ at 0.5 coverage. Chemical Physics 1996, 205, 73-89.

(35) Scholtz, R.; Floss, G.; Saalfrank, P.; Fuchsel, G.; Loncaric, I.; Juaristi, J. I. Femtosecond-laser induced dynamics of $\mathrm{CO}$ on $\mathrm{Ru}(0001)$ : Deep insights from a hotelectron friction model including surface motion. Physical Review B 2016, 94, 165447.

(36) Scholtz, R.; Lindner, S.; Loncaric, I.; Tremblay, J. C.; Juaristi, J. I.; Alducin, M.; Saalfrank, P. Vibrational response and motion of carbon monoxide on $\mathrm{Cu}(100)$ driven by 
femtosecond laser pulses: Molecular dynamics with electronic friction. Physical Review B 2019, 100, 245431.

(37) Loncaric, I.; Alducin, M.; Saalfrank, P.; Juaristi, J. I. Femtosecond laser pulse induced desorption: A molecular dynamics simulation. Nuclear Instruments and Methods in Physics Research B 2016, 382, 114-118.

(38) Füchsel, G.; Klamroth, T.; Monturet, S.; Saalfrank, P. Dissipative Dynamics within the Electronic Friction Approach: the Femtosecond Laser Desorption of $\mathrm{H}_{2} / \mathrm{D}_{2}$ from Ru (0001). Phys. Chem. Chem. Phys. 2011, 13, 8659-8670.

(39) Hertl, N.; Martin-Barrios, R.; Galparsoro, O.; Larregaray, P.; Auerbach, D. J.; Schwarzer, D.; Wodtke, A. M.; Kandratsenka, A. arXiv preprint arXiv:2103.03005 2021 ,

(40) Pavanello, M.; Auerbach, D. J.; Wodtke, A. M.; Blanco-Rey, M.; Alducin, M.; Kroes, G.-J. Adiabatic Energy Loss in Hyperthermal H Atom Collisions with $\mathrm{Cu}$ and Au: A Basis for Testing the Importance of Nonadiabatic Energy Loss. J. Phys. Chem. Lett. 2013, $\underline{4}, 3735-3740$.

(41) Kroes, G.-J.; Pavanello, M.; Blanco-Rey, M.; Alducin, M.; Auerbach, D. J. Ab Initio Molecular Dynamics Calculations on Scattering of Hyperthermal $\mathrm{H}$ Atoms from $\mathrm{Cu}$ (111) and $\mathrm{Au}(111)$. J. Chem. Phys. 2014, 141, 054705.

(42) Ibarguen Becerra, C.; Crespos, C.; Galparsoro, O.; Larregaray, P. Atomic scattering of $\mathrm{H}$ and $\mathrm{N}$ on $\mathrm{W}(100)$ : Effect of lattice vibration and $\mathrm{T}$ electronic excitations on the dynamics. Surface Science 2020, 701, 121678. 